\title{
José María Arguedas, etnógrafo: campo cultural y mestizaje
}

\author{
José María Arguedas, Ethnographer: \\ Cultural Field and Mestizaje
}

\author{
ENRiQue E. Cortez \\ Portland State University \\ ecort2@pdx.edu
}

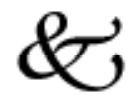

\begin{abstract}
Resumen
En la actualidad, es imposible trazar una historia de la etnología en el Perú sin tomar en cuenta las contribuciones de José María Arguedas. Sin embargo, la antropología de su época atribuyó escasa importancia a su trabajo. El propósito de este artículo es identificar los motivos del reconocimiento tardío y póstumo del aporte de Arguedas. El argumento central es que el concepto de mestizo manejado por la etnografía de Arguedas implicaba una crítica profunda de las prácticas deshistorizantes de la antropología dominante. Interviniendo de manera decisiva en un debate clave en la historia intelectual latinoamericana, Arguedas cuestionó la ideología hispanista del mestizaje de la primera mitad del siglo XX, que entendía tal condición como la realización del ideal de un sincretismo armónico. Retrospectivamente, es posible argüir que su formulación de lo mestizo tiene elementos en común con paradigmas culturales contemporáneos que valorizan la diferencia y el multiculturalismo.
\end{abstract}

Palabras claves: Mestizaje, Etnografía, Valcárcel, Indigenismo, Hispanismo, Giro antropológico 


\begin{abstract}
At present, it is difficult to trace the history of ethnology in Peru without taking into account the contributions of José María Arguedas. During his lifetime, however, his contemporaries in anthropology placed little importance on his work. In this article, I intend to identify the motives behind his belated and posthumous recognition as a contributor to anthropology. The main point I would like to argue is that the concept of mestizo that Arguedas works with in his ethnographies implies an in-depth critique of the dehistoricizing practices of dominant anthropology. By decisively intervening in a key debate of Latin American intellectual history, Arguedas questions early 20th century Hispanist ideology, which understood the condition of mestizaje as a realization of a homogenous, harmonic ideal. In retrospect, it can be argued that his formulation of mestizaje shares elements with contemporary cultural paradigms that place value on diversity and multiculturalism.
\end{abstract}

Keywords: Mestizaje, Ethnography, Valcárcel, Indigeneity, Hispanism, Anthropological Turn

Recibido: 26/4/16 Aceptado: 20/5/16

Cuando en 1975 Ángel Rama publicó su edición de los ensayos etnológicos de José María Arguedas, Formación de una cultura nacional indoamericana, el quehacer del novelista como etnógrafo no tenía la atención que ahora tiene en cualquier trabajo serio sobre su obra literaria. Su revalorización en el campo de la antropología peruana es más tardía, pero lo seguro es que en la actualidad es del todo imposible trazar una historia de la etnología en el Perú sin referirnos al aporte de Arguedas. ${ }^{1}$ ¿Cuál es la razón de que en su campo profesional Arguedas tenga un reconocimiento tardío y póstumo? No parece, como supuso

1 La más detallada historia de la formación de los estudios etnológicos en Perú continúa siendo "Apuntes para una historia de la antropología social peruana: décadas de 1940-1980" de Jorge Osterling y Héctor Martínez. Una reseña de las tendencias teóricas de la antropología peruana lo constituye el libro editado por Carlos Ivan Degregori, No hay país más diverso, donde Arguedas ocupa un lugar importante. Degregori, en el artículo que abre el libro remite la práctica antropológica en Perú hasta la época de la Conquista (2000: 24-30), y es de particular interés su conexión entre el Informe de Uchuraccay y el fin del tipo de antropología culturalista que se estuvo haciendo en Perú (esta reunía tendencias de influencia aplicada, desarrollista, estructuralista, marxista): "En todo caso, termina una manera esencialista de entender a las comunidades y a los pueblos indígenas como reductos congelados de una tradicionalidad ubicada fuera del tiempo y al margen del país" (2000: 49). También resulta útil la retrospectiva de Frank Salomón sobre la etnología andina en la década de 1970, y para las relaciones entre historia y antropología el trabajo de Manuel Burga que cubre de 1987 a 1998. 
Rama, en su introducción al libro mencionado, que el novelista haya opacado al etnólogo (1975: IX); elementos de otra índole, que tienen que ver con la lógica del campo y sus tensiones discursivas y de poder, marginaron el trabajo arguediano, aunque sus aportes hayan sido desarrollados de manera sostenida sin mencionar su nombre, o en otros campos, como ocurrió con el mito de Inkarri, circulado por Arguedas y notablemente influyente en el trabajo de los historiadores de la utopía andina durante la década de $1980 .^{2}$ Este acercamiento, a diferencia de los pocos estudios que abordan el trabajo etnográfico de Arguedas, deja de lado su obra de ficción. La literatura arguediana, lejos de aclarar el tipo de proyecto que su escritura etnográfica desarrolla, actúa como literatura, es decir, constituye un significante lo suficientemente complejo como para postular la existencia de una lectura excluyente o más autorizada. El contrapunto con la literatura, como muestra la seminal interpretación sobre su obra de Alberto Flores Galindo (1992), ha permitido plantear hasta oposiciones en su producción de escritor de ficción y de ensayos antropológicos, plataforma desde la cual lo literario aparece como el discurso de avanzada. Esa dirección interpretativa, por supuesto posibilitada por los diversos registros de la escritura arguediana, ha sido explorada de manera eficaz por Misha Kokotovic (2006). Al destacar el contrapunto mencionado entre ficción y etnografía Kokotovic puede considerar un desfase entre ambos discursos, siendo el literario el género trascendental, y hasta establecer una cronología temática en la producción arguediana bastante operativa si el objetivo es presentar una imagen global del autor de Los ríos profundos. El presente artículo, sin embargo, se detiene un poco antes de las elaboraciones finales, interrogando acerca de qué nos queda del Arguedas etnógrafo, ya no observado desde el prisma de la literatura, sino desde las tensiones ideo-metodológicas de su campo disciplinario. Este Arguedas etnógrafo, estudiado desde sus escritos no ficcionales, más que un intelectual "colonizado" salvado por su literatura, como ha propuesto Nelson Manrique (1999: 98), se plantea como el signo de una trayectoria personal, marcada por su experiencia de intelectual periférico, negociando un espacio de saber entre metodologías y discursos, a veces del todo extraños a su experiencia e historia. Desde esta perspectiva, la indagación del etnógrafo Arguedas sobre lo mestizo es también una intervención poderosa en un campo todavía joven, como fue la antropología en el Perú en las décadas de 1950 y 1960, una disciplina que nació como un último aletazo del indigenismo.

2 Hiroyasu Tomoeda muestra la importancia del mito, en versión de Arguedas, en los libros de Alberto Flores Galindo, Buscando un Inca, de 1987, y en el de Manuel Burga, Nacimiento de una utopía, de 1988. Ambos libros, además, han sido muy importantes en las ciencias sociales, no sólo peruanas. 
Como han indicado Jorge Osterling y Héctor Martínez en sus "Apuntes para una historia de la antropología social peruana: décadas de 1940-1980”, el impulsor más importante de la etnología peruana fue Luis E. Valcárcel, quien como una empresa personal fue logrando, gracias a su trabajo en áreas estratégicas del Estado peruano, crear espacios institucionales para la antropología en el Perú. ${ }^{3}$ Entre estos espacios tenemos primero al Museo Nacional, del que fue director desde 1931 hasta 1964, y en el que formó una sección dedicada a la antropología, "encargado de estudiar al hombre y la cultura del periodo precolombino" (Osterling y Martínez, 1985: 37); y, en segundo lugar, las varias instituciones que creó entre 1945 y 1947, época en que ejerció de ministro de Educación. En este último periodo destacan sobre todo el Instituto de Estudios Etnológicos, afiliado al también en ese entonces reciente Museo de la Cultura Peruana (creado en 1945), y el Instituto de Etnología y Arqueología (creado en 1946), como parte de la Facultad de Letras de la Universidad Nacional Mayor de San Marcos. Este será el primer lugar de enseñanza de los estudios etnológicos y al que se remonta el origen de la disciplina en el Perú (Osterling y Martínez, 1985: 37 y 38).

Pero Luis E. Valcárcel no sólo fue el fundador de la antropología en el Perú; en el panorama de las ideas del siglo XX peruano no podemos dejar de lado su influyente libro, Tempestad en los andes, publicado en 1927 y central en la formulación del indigenismo peruano. Su actividad como etnólogo e indigenista, en consecuencia, nunca estuvo escindida; al contrario, en 1946, "merced a su influencia en los círculos gubernamentales más altos, logra el establecimiento del Instituto Indigenista Peruano" (Osterling y Martínez, 1985: 37), como parte del aparato burocrático del Estado. Entre las funciones del instituto tendremos el estudio de las poblaciones aborígenes, sus condiciones de vida, la sugerencia de leyes y resoluciones que aseguren un mayor bienestar de estas poblaciones, la colaboración con instituciones nacionales y extranjeras y la publicación de una revista: "estas funciones le permiten [al instituto] la realización de un conjunto de investigaciones, y la participación de acciones declaradamente indigenistas, por sí mismo y en colaboración con el Proyecto Perú-Cornell, el

3 La institucionalización del indigenismo en el Perú, como nos recuerda Degregori, se construyó bajo la influencia de la experiencia mexicana, "México aparece como punto de referencia y contraste” (2000, p. 38); para Javier Ávila Molero sería un error considerar sólo la iniciativa personal de Valcárcel, habría que considerar "la fuerte influencia del indigenismo mexicano posrevolucionario" (2000, p. 418). Es cierto que no se puede negar el contexto ideológico que influenciaron las iniciativas de Valcárcel, pero si éste no hubiera tenido un cargo tan medular en la administración del Estado de entonces, ministro de Educación, otra sería la historia de la antropología peruana. 
Programa Puno-Tambopata, y el Proyecto de Integración y Desarrollo de la Población Indígena” (Osterling y Martínez, 1985: 40).

La fuerte conexión entre indigenismo y teoría del desarrollo, que se desprende de las prácticas del instituto, no han sido para nada ajenas a la formación de la antropología en el Perú. ${ }^{4}$ El mismo Valcárcel, informa al respecto:

[...] desde la etnología fue vertebrándose el nuevo indigenismo. Con las nuevas disciplinas éste [el indigenismo] asumió un carácter científico y práctico, pues las opiniones de los etnólogos comenzaron a ser consideradas como la condición previa para cualquier plan destinado a mejorar las condiciones de vida de la población aborigen. La perspectiva indigenista se incorporó a los fines del desarrollo a partir de 1946 con la fundación del Instituto Indigenista Peruano [...] los etnólogos egresados de San Marcos colaboraron con los técnicos del Estado dándole a sus apreciaciones un enfoque científico social. (1985: 23 y 24)

La aparente contradicción entre el indigenismo y la perspectiva aculturadora de la antropología aplicada de la década de 1950 no era del todo opuesta. Ambas posiciones tenían en común la deshistorización de lo indígena (Archibald, 1988: 8), como una mirada pasatista en el caso del indigenismo y como una mirada desarrollista y modernizadora en el caso de la antropología aplicada, para la cual la cultura e historia indígena representaban el subdesarrollo. Ambos discursos también eran esencialistas (de lo indio el primero, del método el segundo); y ambos, a su vez, fueron una acción desde afuera sobre lo indígena, como ya José Carlos Mariátegui aclaró en sus Siete ensayos, a propósito de la literatura indigenista: "la literatura indigenista no puede darnos una versión rigurosamente verista del indio. Tiene que idealizarlo y estilizarlo. Tampoco puede darnos su propia ánima. Es todavía una literatura de mestizos. Por eso se llama indigenista y no indígena. Una literatura indígena, si debe venir, vendrá a su tiempo. Cuando los propios indios estén en grado de producirla" (1979: 221). ${ }^{5}$ La conversión del indigenismo a la teoría del desarrollo aparece así como la salida pragmática ante el entrampamiento que suponía el incaismo, como espacio utópico, que fue el elemento central del discurso indigenista de las décadas de 1920 y 1930. Esta nueva fase del indigenismo -conectado a la

4 Sobre la teoría del desarrollo en los estudios etnológicos peruanos es central el artículo de Priscilla Archibald (1998). También el trabajo de Misha Kokotovic (2006) amplía algunos aspectos. Javier Ávila Molero (2000) revisa el trabajo de las instituciones vinculadas a la teoría del desarrollo desde el Programa Perú-Cornell hasta los Organismos No Gubernamentales.

5 Sobre indigenismo hay mucho escrito. Me parece claro el uso que hace Tomoeda (2006: 167 y 68) del término, quien resume los argumentos sobre el tema de William Rowe y Flores Galindo. En general, es la misma definición que en 1927 hizo Mariátegui, en Siete ensayos. Para la literatura peruana es central el trabajo de Tomas Escajadillo (1994). 
etnología, con instituciones en el Estado y apoyo norteamericano- se convertirá en el rector de la política cultural en la década de 1970, durante el gobierno militar del general Juan Velasco Alvarado. Este nuevo revival del indigenismo político, correlativo al indigenismo 1 -de finales del siglo XIX hasta la década de 1920- como ha llamado Mirko Lauer a esta etapa, no está del todo divorciado del indigenismo cultural ("denominado indigenismo 2", 1996: 46), sino que tiene su origen en ese abrazo entre indigenismo y teoría del desarrollo en el que se fundaron los estudios etnológicos en el Perú.

Es en este entramado que la intervención de Arguedas cobra todo su sentido. Como sabemos, Arguedas fue de los primeros estudiantes en matricularse en el programa doctoral de etnología en la década de 1950. Para ese momento ya era conocido como escritor y llegó al campo con un capital simbólico construido a lo largo de los años a través de los periódicos, de sus novelas y, no debe olvidarse, como promotor cultural. En este punto tiene particular importancia las actividades que la peña Pancho Fierro desarrolló durante tres décadas desde su inauguración en 1936. Esta peña, lugar de encuentro bohemio y diálogo intelectual, albergó también

[...] una colección de arte popular andino formada por piezas traídas de los viajes de Alicia Bustamante, su hermana Celia y José María Arguedas, al interior del país donde adquirían máscaras, toros de Pucará, retablos, mates burilados. La colección, producto de intensas búsquedas, constituyó un hecho excepcional porque, en aquel tiempo, lo producido por los serranos carecía de valor en el medio limeño. (Bernabé, 2006: 379)

La influencia del grupo de escritores y artistas afiliados a la peña Pancho Fierro ha sido determinante en la historia cultural peruana. La presencia de lo popular en medio de la alta cultura limeña se debió a la especial sensibilidad que las hermanas Bustamante tuvieron por las manifestaciones indigenistas. Alicia, quien fuera pintora y discípula de José Sabogal, y Celia, la primera esposa de Arguedas, permitieron al escritor y futuro etnólogo una plataforma alternativa al circuito hispanista pero no marginal, si recordamos que el indigenismo poco a poco, a través del trabajo de Valcárcel, fue ocupando un lugar institucional central en el aparato del Estado.

Arguedas, en consecuencia, no carecía de interés y trayectoria cuando en 1952, estudiante de San Marcos, en su informe sobre el Primer Encuentro Internacional de Peruanistas introdujo el tema del mestizo en el contexto del indigenismo, en radical desacuerdo con las propuestas de Valcárcel. Este distanciamiento intelectual de su profesor de San marcos, no ha sido hasta ahora destacado suficientemente, pero es clave para entender su poca circulación en el ambiente académico de la década de 1960, como se verá después. De 
hecho, en el excelente estudio de Marisol de la Cadena sobre el mestizaje indígena, Arguedas no tiene ningún lugar a no ser como un elemento afiliado al "differentialist racism" de la propuesta indigenista de Valcárcel (2000: 168). ${ }^{6}$ (6) Sin embargo, como explica Rama, el distanciamiento de Arguedas de las posiciones intelectuales de Valcárcel le permitirá articular su propia línea interpretativa: "contrariamente a la opinión negativa de Luis Valcárcel, que hiciera escuela, afirmará que el mestizo representa una clase social real, existente y numerosa, que ya puede caracterizarse con bastante precisión, salvo que no ha sido estudiada a pesar de ser elemento clave 'de las posibilidades y el destino del país"' (1975: XVIII). Dentro del esquema del indigenismo, el estudio del mestizo suponía dos movimientos: desprenderse de la idea del indio como agente de cambio social y, lo fundamental, dejar atrás la idea nostálgica de recuperar el incanato, reconociendo, al contrario, "la cultura india mestizada poshispánica, lo que implicaba certificar una extraordinaria capacidad de adaptación por parte del pueblo quechua a lo largo de la Colonia" (Rama, 1975: XVIII). La crítica de Arguedas a Valcárcel muestra ecos de la idea de vivencia, que toma del pensamiento de Wilhen Dilthey, cita explicita en "La sierra en el proceso de la cultura peruana” (Arguedas, 1975: 9), articulo originalmente aparecido en el diario limeño La prensa en 1953. Esta idea de vivencia, como expresión de la experiencia, es la que apoyará en contra de la descalificación que sobre el mestizo hizo el fundador de la etnología peruana:

Valcárcel representa la corriente pesimista acerca del mestizo. Pero toda persona que haya vivido en muchas ciudades y aldeas de la sierra, sabe por propia experiencia que el mestizo no representa solo 'un borroso elemento de la clase media', sino la mayoría y, en algunos casos, como en los pueblos del valle alto del Mantaro (provincias de Jauja y Huanca-

6 Esta afiliación con Valcárcel evita, asimismo, que De la Cadena incorpore en su estudio el aporte arguediano sobre el mestizo. Pero en más de un sentido, podemos ver en sus aportes sobre la transformación del concepto de mestizaje, un recorrido simétrico al de Arguedas, que va desde una posición elitista que suponía altos grados de asimilación hacia el uso más flexible que hacen del término las clases populares cusqueñas. Dice De la Cadena: "For working-class cusqueños self-identification as mestizos implies changing social conditions, but not cultures, as I had been used to thinking [...] Within this process, a de-Indianizing individual can be mestizo and indigenous at the same time. This individual (the indigenous mestizo) considers herself neto and thus familiar with practices deemed extraneous to the dominant culture and at the same time understands practices that are perceived as belonging to the dominant national formation" (2000: 30, énfasis de la autora). Para resumir, es posible observar que lo que Arguedas entendía como mestizo es lo que De la Cadena, al final de la década de 1990, denominará "indigenous mestizo", una forma de especificar el sentido de la identidad cultural de una población concreta que utiliza el término mestizo para autorrepresentarse. Tal ampliación del sentido del término mestizo, solo será posible para De la Cadena a partir de su experiencia de trabajo de campo, experiencia que también fue decisiva en la formulación de Arguedas. 
yo), la totalidad de la población de estas ciudades y aldeas. (Arguedas, 1975: 3)

Lo que sigue, por parte de Arguedas, es la propuesta de un posicionamiento metodológico para el reciente campo de la antropología peruana: "El estudio del mestizo es uno de los más importantes de los que la antropología está obligada a emprender en el Perú. Hasta el presente solo se han escrito ensayos que tienen reflexiones sobre el problema; no se ha cumplido aún un verdadero plan de investigación en contacto con el hombre mismo" (Arguedas, 1975: 2). Lo interesante de Arguedas es que lleva dos miradas al campo de la etnología de entonces. Por un lado, habla de estudiar al hombre mismo, en su coordenada mestiza, en términos de antropología aplicada; por otro lado, ese énfasis en lo mestizo es un intento de atender el proceso histórico también implicado en este actor. El énfasis en la historia es el elemento principal que diferencia la escritura etnográfica de Arguedas. Como ha mostrado Archibald, tal historicidad va en dirección contraria a las prácticas de la antropología aplicada, que tuvo en la hacienda de Vicos su laboratorio, como parte del Proyecto Perú-Cornell. ${ }^{7}$

Lo más resaltante del trabajo arguediano en el campo de la etnología serán sus estudios sobre Puquio y sobre el Valle del Mantaro, espacios donde el mestizo como actor de cambio tendrá una centralidad, antes de Arguedas, nunca concedida por las ciencias sociales. ${ }^{8}$ Antes de revisar estos trabajos, quiero detenerme en un aspecto crucial para entender la importancia y actualidad de este debate. ¿Qué entiende Arguedas por mestizo? En "El complejo cultural en

7 Tal contraste entre el trabajo de Arguedas y los etnólogos de la época, extranjeros y peruanos, es muy explícito en la publicación de los primeros trabajos del proyecto Perú-Cornell, titulado Estudios sobre la cultura peruana actual, paradójicamente con prólogo de Arguedas y donde publicará su estudio sobre Puquio. Sobre la importancia del proyecto Perú-Cornell, y su trabajo en la hacienda Vicos, ver Osterling y Martínez (1985), así como las notas de Valcárcel (1985). La biblioteca de Cornell University tiene en línea materiales que informan del trabajo de sus profesores y estudiantes en la hacienda Vicos: http://rmc.library.cornell.edu/EAD/pdf_guides/ RMA01529_A.pdf

8 No podemos olvidar, por supuesto, su trabajo comparativo entre las comunidades campesinas de España y las del Perú, investigación presentada como tesis doctoral en San Marcos. Aparte de ser un trabajo innovador, y con grandes aciertos para la etnología comparada y los propios estudios en España, es un gesto de lo más significativo para la etnología latinoamericana, y puede leerse como subversiva del usual trayecto de la geopolítica del saber: de norte a sur, del atlántico al pacífico. Sobre el valor de la etnografía en sí, Fermín del Pino, hace una evaluación que destaca sus aciertos y la excepcionalidad de este trabajo. En relación a los etnólogos de la época (supuestamente mejor formados que Arguedas) que estudiaban España, dice Del Pino "Da la impresión [...] que Arguedas comprende mejor que sus colegas anglosajones el significado peculiar de instituciones como el compadrazgo (que no lo encuentra Arguedas en Sayago), el poder particular de la iglesia y de la burocracia local en el pueblo, la separación rígida de los sexos y las normas relativas al sexo [...] Arguedas sabe preguntarse por las peculiares condiciones de vida castellanas" (1995: 47). 
el Perú”, texto originalmente aparecido en 1952, Arguedas define al mestizo en términos culturales, ya no raciales y morales como el indigenismo:

\begin{abstract}
Durante siglos, las culturas europeas e india han convivido en un mismo territorio en incesante reacción mutua, influyendo la primera sobre la otra con los crecientes medios que su potente e incomparable dinámica le ofrece; y la india defendiéndose y reaccionando gracias a que su ensamblaje interior no ha sido roto y gracias a que continúa en su medio nativo; en estos siglos, no sólo una ha intervenido sobre la otra, sino que como resultado de la incesante reacción mutua ha aparecido un personaje, un producto humano que está desplegando una actividad poderosísima, cada vez más importante: el mestizo. Hablamos en términos de cultura; no tenemos en cuenta para nada el concepto de raza. Quienquiera puede ver en el Perú indios de raza blanca y sujetos de piel cobriza, occidentales por su conducta. (Arguedas, 1975: 2)
\end{abstract}

Esta breve cita abre varias vías de argumentación. La primera tiene que ver con el enfoque histórico, pues la presencia del mestizo le permite postular a Arguedas una historia transcultural, de mutuas influencias, no exenta de violencia. Por eso, Arguedas utiliza el término 'reacción'. La segunda línea explorada es la sincrónica: desde la experiencia actual de las grandes migraciones del campo a la ciudad, el mestizo, aparecerá desplegando una actividad "poderosísima”. En tercer término, Arguedas precisa que no habla desde la raza, o, quizá, sería mejor decir que no sólo habla desde la raza, porque el término mestizo tiene esa connotación racial como parte de su sentido. En un gesto por resignificar el término, Arguedas enfatiza el carácter móvil que tiene el concepto y por ello, más adelante, dirá que "hay infinidad de grados de mestizaje; que es muy distinto el que se forma en los pueblos pequeños de la sierra y el que aparece en las ciudades" (Arguedas, 1975: 3). Con esto el autor de Los ríos profundos no está haciendo más que presentarnos un término complejo y con alcance histórico, que intenta, a su vez, describir una realidad compleja como es el de las relaciones entre la cultura europea y la indígena desde la época de la conquista. En este punto diremos que Arguedas está pensando fuera de la caja de las coordenadas de la antropología de la época, porque lo está haciendo a partir de una perspectiva de historia cultural, construyendo, a la vez, una línea de pensamiento político donde ese actor, definido históricamente como mestizo, tendría un rol protagónico. Con la distancia que da el tiempo, podemos decir que no se equivocó en absoluto, y el rol de aquellos sujetos representados en el término mestizo es central en la actualidad cultural, económica y política del Perú.

Sin embargo, la calidad compleja del planteamiento de Arguedas sobre lo mestizo no ha sido apreciada, precisamente por ese excedente de complejidad 
que haría parecer al término poco operativo en términos descriptivos desde alguna metodología de las ciencias sociales. Esto quizá se deba a un malentendido, surgido en una lectura que busca continuidad en las posiciones discursivas, paralelas a una coherencia biográfica (por cierto, solo lograda por el biógrafo). Nelson Manrique, por ejemplo, señala, apoyándose en la lectura que de Arguedas hizo Alberto Flores Galindo en Buscando un Inca, que "el discurso del mestizaje en la obra de Arguedas no es lineal ni unívoco. Por el contrario, está atravesado de tensiones y, en determinados momentos, profundas contradicciones" (Manrique, 1995: 78). Las "profundas" contradicciones que encuentra Manrique tienen que ver con lo que él lee como una propuesta de integración nacional armónica en la obra de Arguedas y desde la cual lo mestizo aparece como algo problemático: "Parecería [...] que la noción de mestizaje, elaborada principalmente a partir de los estudios de Arguedas sobre el valle del Mantaro, habría sido el punto de llegada de su búsqueda de una integración nacional armónica" (Manrique, 1995: 79). Esta idea de contradicción es la que Flores Galindo propondrá al analizar el discurso que Arguedas pronunció al recibir el Premio Inca Garcilaso de la Vega en 1968, un año antes de su suicidio. En este discurso, como es de todos sabido, Arguedas afirmará autobiográficamente su condición de bilinguie linguístico y cultural, pues en su experiencia vital tanto el quechua como el español han coexistido no en términos de fusión armónica sino como una realidad heterogénea. Como Flores Galindo interpreta la propuesta de mestizaje de Arguedas en términos de homogeneidad y armonía es entendible que ubique una contradicción en el discurso arguediano. Es lo que repetirá Manrique al denunciar las "profundas contradicciones". Por supuesto, como nos recuerda Kokotovic, Manrique también cuestiona de esa manera la presencia de elementos de la teoría del desarrollo en los estudios arguedianos (2006: 115 y 116). Según Kokotovic, Arguedas recién podrá separarse de la antropología aplicada en su trabajo literario, a partir de la publicación de su novela Los ríos profundos, porque "un código cultural andino moldea la historia en Los ríos profundos, permitiéndole a Arguedas imaginar alternativas al status quo semi-feudal más radicales que las limitadas opciones esbozadas en sus artículos antropológicos de los años 50" (2006: 98). En mi lectura, lo importante no es identificar los grados de "corrupción colonizante" de su tarea como etnógrafo, sino destacar su trabajo con lo mestizo en ese preciso contexto desarrollista. Quizá se pueda hablar de etapas del concepto y trabajar con alguna de ellas si es conveniente para algún acercamiento. En mi estudio sobre lo mestizo, limitado a la obra etnográfica de Arguedas, el término se me presenta saludablemente complejo y tal vez no operativo para una metodología rígida, pero que se revela como un aporte al pensamiento sobre la cultura peruana. El trabajo de resemantización que Arguedas hace con el término, rescatándolo 
de la utopía hispanista, tiene un potencial hasta ahora no atendido, no porque una parte del trabajo de Arguedas siga opacando otras áreas, como metaforiza Rama en la introducción a su edición de los ensayos antropológicos, sino porque sus lectores siguen leyéndolo en coordenadas disciplinarias sin considerar que estamos frente a un pensador de la cultura y la política peruana. Es un poco la línea en que lo recuperará al inicio del presente siglo Carlos Iván Degregori:

José María Arguedas es la figura emblemática de una de las posibilidades de tránsito, y de frustración. De manera confusa, intuitiva, desgarrada, tanto en sus trabajos antropológicos como literarios, avizora la posibilidad de un 'nosotros diverso' más allá de los desgarramientos coloniales y del mestizaje homegenizante propuesto por el poder [...] Arguedas logra intuiciones que lo convierten en precursor de una interculturalidad sustentada teóricamente y popularizada recién 10 o 15 años después de su muerte. (2000: 44 y 45)

No obstante, la recuperación de Degregori, no revisa que la posibilidad de ese "nosotros diverso" y la alternativa al mestizaje homogenizador se formula en la manera en que Arguedas conceptualizará su propuesta de mestizaje. Se trata de la apropiación de un término connotado políticamente por la ideología del hispanismo de las primeras décadas del siglo XX, y esta es una de las razones por la que alguien tan atento como Cornejo Polar descartará el uso de mestizaje de Arguedas. Con esto no quiero decir que el alcance de la propuesta hispanista, que tuvo en el Inca Garcilaso su más acabado modelo de mestizaje, como una utopía armónica, no haya sido un elemento que connotaba el término mestizo. Lo que digo es que Arguedas lo usa de otra manera y hacer distancia del uso del hispanismo no debería significar un distanciamiento de la propuesta de Arguedas. Veamos la lectura de Cornejo Polar:

[...] la visión rivagueriana del Inca es decidida y hasta fanáticamente aristocratizante pero, restándole sus excesos, estableció un sólido estereotipo cuya trama, como se ha visto, tiene dos grandes articulaciones: la que insiste en que el Inca Garcilaso es símbolo de un mestizaje armónico, y por ese camino, símbolo de la peruanidad, y la que subraya la excepcionalidad de tal mestizaje por ser doblemente nobilario. Hay que convenir que la primera de estas interpretaciones ha calado profundamente en la conciencia de vastos grupos sociales, inclusive en sectores del pensamiento indigenista que, directa o indirectamente, apuestan a favor de un mestizaje integrador, como podría ser el caso de Uriel García. (1994: 106)

Este tipo de mestizaje, entendido como una experiencia armónica, es al que alude Flores Galindo. ${ }^{9}$ Tal idea circulada por José de la Riva Aguiero, no po-

9 No deseo parecer injusto con la lectura de Flores Galindo, una de las más interesantes y plena 
día ser para nada la de Arguedas, quien claramente también tomó distancia de los hispanistas y hasta del Inca Garcilaso de la Vega, a quien opondrá la figura de Guaman Poma de Ayala. En efecto, el 17 de diciembre de 1939 Arguedas publicó en La prensa un fragmento de la obra de Guaman Poma, a partir de la primera edición facsimilar que en 1935 el Instituto de Etnología de París, hizo de la Nueva coronica y buen gobierno. Este texto, titulado "Doce meses", tiene un subtítulo que informa acerca de las primeras incursiones de Arguedas como traductor. Dice el subtítulo: "Un capítulo de Guaman Poma de Ayala. Versión de las frases Kechwas e interpretación del estilo" (1989: 28). Antes de proponer su versión del calendario agrario andino, que es también una suerte de sumario de las costumbres y festividades, Arguedas nos presenta en algunos párrafos al cronista indio, diciendo que su obra "ha venido a rectificar y completar la obra de todos los cronistas anteriores, especialmente a Garcilaso" (1989: 28).

Este deslinde con el Inca Garcilaso de la Vega es para Arguedas, fundamentalmente, un deslinde con sus lectores, es decir, con los hispanistas que le habían otorgado un simbolismo que él cuestiona. En "El indigenismo en el Perú", texto escrito para un coloquio de escritores realizado en Génova, 1965, Arguedas informa que "Garcilaso es interpretado por Riva-Aguiero como un símbolo del mestizaje imperial: es excelso porque es el fruto del cruce de dos razas en el plano más elevado: el de la aristocracia; y Garcilaso, el Inca católico, defiende y magnifica las virtudes del régimen imperial incaico" (1989:10). Nótese que la interpretación arguediana del simbolismo otorgado por Riva Aguiero al Inca es la misma que décadas después desarrollará Cornejo Polar, como se observa en la cita arriba transcrita. Esto demuestra lo atento que estaba Arguedas al usar un término como mestizo, políticamente afiliado al hispanismo, que buscaba, además, como ha resaltado Cornejo Polar, "imaginar la nación armónica a partir de una figura inaugural como el Inca", a partir de la idea de homogeneidad cultural que en el caso del mestizaje no sólo insiste "en la convergencia pacífica y constructiva de las dos 'razas' que entraron en contacto con la Conquista,

de intuiciones que se han hecho de Arguedas desde la historia y que resultará fundamental para su recuperación desde las ciencias sociales (ver, por ejemplo, la lectura de Gonzalo Portocarrero en Racismo y mestizaje). Pero lo mejor del acercamiento de Flores Galindo a la obra de Arguedas se encuentra en Dos ensayos sobre José María Arguedas, texto posterior a la redacción de Buscando un Inca. Por cierto, Buscando un Inca es el texto que marca la línea interpretativa de Manrique. En Dos ensayos, por ejemplo, al referirse al proceso de elaboración de El zorro, la novela póstuma de Arguedas, Flores Galindo hace una conexión importante entre antropología y literatura, precisamente, como efecto de las tensiones disciplinarias en las que se desarrolló su actividad: "Empezó desde la vertiente antropológica, del lado que partía el mundo andino. Sea por la desavenencia con los intelectuales, o por descubrir que esos instrumentos [los de la antropología aplicada] no eran útiles para entender lo que pasaba [el cambio cultural], el proyecto antropológico se fue convirtiendo en novela" (1992: 25). 
sino que sustituye el significado bélico de esta palabra para expresar con ella la fusión amorosa de ambas [...] Nacida del amor y no de la destrucción y la muerte, la patria resulta ser suma y unimismamiento de lo vario y distinto. El mestizaje es su representación preclara" (1994:104 y 105).

Arguedas también hará distancia de Raúl Porras Barrenechea, a quien califica de continuador de Riva-Aguiero, por publicar un estudio "injurioso y panfletario contra el cronista indio Felipe Guaman Poma de Ayala" (1989:10). Al contrario, una lectura positiva de la Nueva coronica como la que hace Julio C. Tello garantizará la simpatía ideológica de Arguedas: "Tello recibe con evidente regocijo el hallazgo y la publicación de la obra de Guaman Poma de Ayala; considera la Nueva Crónica y Buen Gobierno (sic) como el testimonio más importante para el estudio de la Colonia y del Imperio, mientras sus contemporáneos, a quienes nos hemos referido, guardan silencio y Porras califica al cronista como a un indio resentido y un autor folklorico" (1989:11).

Este posicionamiento de Arguedas, como hemos señalado, se inicia en 1939 con su traducción del cronista indio. Al momento en que el escritor empieza a producir como etnólogo sus posiciones también habían madurado, de modo que su noción de mestizo no surge del aire y de la ingenuidad como parecerían entender algunos de sus lectores. Al contrario, Arguedas retoma el término mestizo del discurso hispanista y lo resemantiza, porque es un término necesario por sus conexiones históricas y porque, además, mucha gente se autorrepresentaba con este término. De igual modo, le parece necesario el término indio cuando la tendencia de la antropología desarrollista buscaba llamarlos campesinos (Archibald 1998, p. 10), borrando las marcas de cultura e historia: "Al hablar de la supervivencia de la cultura antigua del Perú nos referimos a la existencia actual de una cultura denominada india que se ha mantenido, durante siglos, diferenciada de la occidental. Esta cultura, a la que llamamos india porque no existe otro término que la nombre con la misma claridad, es el resultado de un largo proceso de evolución y cambio" (Arguedas 1975: 1 y 2 , énfasis del autor).

En esta atención por las palabras se expresa, por supuesto, el narrador y poeta que también fue Arguedas. Como bien ha señalado Juan Javier Rivera Andía, un estudio sobre el antropólogo Arguedas no debe dejar de lado la diferencia personal que el escritor trajo a su quehacer profesional de etnólogo: "creemos que el sentido estético es un concepto fundamental para entender la obra etnológica de Arguedas" (2004: 263). Para Rivera Andía este sentido estético se aprecia en su sensibilidad artística que le permitía "acercarse mejor al arte de otros pueblos: a sus danzas, a su música, a su literatura, a los símbolos densos que ella usa para expresar sus obsesiones, sus miedos y sus fascinacio- 
nes colectivas" (2004: 266). ${ }^{10}$ Este trabajo como folklorista, como se denominó a sí mismo Arguedas, y que Rama prefiere llamar sociología del arte, ya que el folklorismo fetichiza los productos de una cultura (Archibald 1998:19), muestra también la atención que el escritor mostró por las palabras. De allí que su labor como traductor de poemas, canciones quechuas y particularmente su trabajo con el manuscrito de Huarochirí, sea reclamado por Rodrigo Montoya como una parte significativa del corpus de su obra etnográfica (1991: 21 y 22).

El cuidado con las palabras está en relación directa a su experiencia como escritor, pero también a su experiencia bilinguie. Para Estelle Tarica, esto se expresará como un conflicto linguiístico y tendrá dos soluciones. La primera, que ella denomina "la poética de la mistura", se presenta como la convivencia del español y el quechua en un mismo contexto linguístico. La segunda, que ella denomina "la poética de la traducción" se expresa de manera eficaz en Los ríos profundos (2006: 15-17). Nos dice Arguedas: "Si hablamos en castellano puro, no decimos ni el paisaje ni nuestro mundo interior; porque el mestizo no ha logrado todavía dominar el castellano como su idioma y el kechwa es aún su medio legitimo de expresión" (1989: 26). Se trata de un impasse que el autor tiene con el lenguaje en su intento de comunicar su interior que es personal y cultural. Este impasse ocurre, según la propuesta de Tarica, porque Arguedas quiere comunicar no solamente "la visión o la voz indígena, sino también la experiencia cambiante de su yo bilinguie” (2006: 18). Continúa Arguedas:

Y de ahí ese estilo de Agua, del que un cronista decía en voz baja y con cierto menosprecio, que no era ni kechwa ni castellano, sino una mistura. Es cierto, pero sólo así, con ese idioma, he hecho saber bien a otros pueblos, del alma de mi pueblo y de mi tierra. Mistura también y mucho más, es el estilo de Guaman Poma de Ayala; pero si alguien quiere conocer el genio y la vida del pueblo indio de la Colonia, tiene que recurrir a él. (1989: 26)

La aproximación de Tarica argumentará que la "mistura" será una poética de tránsito hacia la traducción, pero también la identificará, siguiendo a William Rowe, como una transferencia a la linguística del concepto de mes-

10 Creo, como Rivera Andía, que tal sentido estético es importante, pero hay que tomarlo con pinzas. Tomoeda ha mostrado que Arguedas, en su estudio sobre Inkarri, le pone un poquito de 'justicia poética' a sus versiones del mito, presentando el retorno de la cabeza como una parte constituyente y central del mito cuando en realidad es sólo una variante puquiana que contrasta con otras versiones del mito recogidas hasta ahora: "hablando teóricamente, la versión no tiene ninguna posición privilegiada dentro de[l] ciclo de Inkarri y su mensaje no queda en la misma, sino en su relación con otras versiones y otros tipos del mito" (2006: 182 y 83). Lo cierto es que también hubo un ambiente adecuado donde la idea de la cabeza que busca el cuerpo decapitado del inca tuvo acogida. En la década de 1970, los historiadores Franklin Pease y Henrique Urbano, retomaron la propuesta de Arguedas. (Tomoeda, 2006: 179) 
tizo: "La idea de mezclar los dos idiomas es una transferencia al campo de la linguiística de la noción de lo mestizo. Es decir, del individuo que representa el conflicto de las dos culturas y que está en el proceso de luchar por crear una síntesis, "una propia personalidad cultural"' (Rowe 1979: 46). Si bien Rowe entiende el mestizaje como una síntesis, comprende también que tal síntesis no es ninguna realidad en Arguedas, en consecuencia, su noción de mestizaje linguiístico será un proceso de lucha de donde no saldrá un tercero que reunirá armónicamente a los dos anteriores, sino un tercero que será completamente diferente, con su "propia personalidad cultural". En el discurso de Arguedas, tal mistura supondrá un elemento de autoconstrucción como autor y sujeto histórico, pues lo situará en una continuidad histórica, imaginándose al lado de la emblemática resistencia andina que representa, en su lectura, la obra de Guaman Poma de Ayala. Con este apunte quiero argumentar que la atención a lo estético en Arguedas significa, de este modo, considerar su formulación de lo mestizo para el campo de la etnografía y el pensamiento sobre la cultura peruana como un ejercicio profundamente autobiográfico. Su formulación conceptual, fiel al postulado de Dilthey del que era lector, se basa en su propia vivencia.

En años recientes, la obra de Arguedas viene siendo revalorada desde los estudios etnológicos. Habiendo caído en desprestigio paradigmas como el funcionalismo o el estructuralismo, el estudio de las redes de significación, a partir de los cuales se definiría la cultura según el trabajo de Clifford Geertz, encuentra en los estudios de Arguedas a un adelantado. Como explican Thomas Krüggeler y Ulrich Muicke, el anthropological turn que generó la propuesta de Geertz, supone principalmente que "la cultura deja de ser el modo de vivir para ser definida como el modo de ver el modo de vivir [...] Geertz no deja de lado el mundo material, sino que constata simplemente que el actuar y los resultados y productos del actuar tienen significados específicos según las diferentes culturas" (2001:13). De modo que lo que interesará serán las interpretaciones, la manera en que el hombre crea su mundo como un "tejido de significados" (Krüggeler y Mücke, 200: 13).

En los casos de Puquio y el Valle del Mantaro, Arguedas analizará la situación particular que cada caso representa a partir de una aproximación histórica. La diferencia de Puquio, la explicará con las transformaciones de producción que una vía de comunicación con la costa y Lima introdujo a principios del siglo XX. En el caso del Valle del Mantaro, la referencia a la historia nos remontará desde el estatus especial que tuvieron los Wankas en la época de la conquista, cuando fueron aliados de los españoles contra la panaka inca de Quito, hasta el hecho de convertirse en pueblo de indios cuando la actividad minera, que se 
realizaba en otros lugares, despobló el área de españoles. A este trabajo con la historia, que Priscilla Archibald ha considerado central para diferenciar la tarea arguediana de sus coetáneos, se suma el hecho del análisis mismo que dará un lugar muy importante a las percepciones. ${ }^{11}$ Rivera Andía, quien ha llamado la atención sobre este punto, nos recuerda que si bien el trabajo de Arguedas, coincide con el de los antropólogos de la época, al estudiar el cambio cultural y al explicar la sociedad estudiada desde la geografía, la historia y la economía, lo notable en él es cómo analiza el cambio cultural, el cual se manifiesta en las percepciones y las relaciones que tienen de ellos mismos los miembros de una comunidad: "La particularidad más notable de las consideraciones de Arguedas al respecto se encuentra en los factores con que relaciona el cambio cultural. Arguedas vincula este cambio con la percepción del otro presente entre los miembros de una sociedad afectada. Así, a una determinada forma de percibir al otro le corresponde un tipo determinado de cambio cultural" (2004: 283). En consecuencia, a mayor separación entre indios y señores, tendremos una sociedad más jerarquizada, con negativos resultados para la conservación de la cultura y el desarrollo económico de la sociedad. Ejemplo de esto serían algunos ayllus de Puquio. Al contrario, a mayor presencia de mestizos (menos señores e indios) el otro será percibido en términos más cercanos y habrá un ambiente más igualitario. Es el caso del Valle del Mantaro (Rivera Andía, 2004: 284). Entre estos dos espacios, Rivera Andía introduce la mención de un tercer espacio que sería Chimbote, y que Arguedas no asimilará a ninguno de los anteriores, atento a los significados específicos. El caso de Chimbote, entendido como caos y desorden por Rivera Andía, no constituyó en rigor una etnografía, pero sí una novela, El zorro de arriba y el zorro de abajo, que incluye, como toda la obra literaria de Arguedas, mucha información etnográfica. ${ }^{12}$

Un punto importante en el análisis del cambio cultural para Arguedas será la valoración ética al que someterá al cambio cultural. Cuando las manifestaciones culturales de una cultura en proceso de cambio se reducen al espa-

11 "This attention to history is one of the distinguishing features of Arguedas's anthropological production. Many of the essays in Formación de una cultura nacional indoamericana are historical narratives" (9).

12 Sobre El zorro de arriba y el zorro de abajo se ha escrito muchísimo en los últimos años. Una de las más importantes críticas es la de Alberto Moreiras, quien lee la novela como una prueba en contra de la propuesta de transculturación de Rama. En todo caso, esta es una crítica al concepto de Rama, más que una lectura atenta del texto arguediano, que también puede ser 'usado', término utilizado por Moreiras al describir su lectura de la novela, para argumentar otras cosas. Ver nuevas lecturas sobre este texto y la obra arguediana en José María Arguedas: hacia una poética migrante (2005). Flores Galindo desarrolla algunas consideraciones en torno al proyecto antropológico de Arguedas en Chimbote, financiado por la Universidad Agraria la Molina, que originará la novela. Al respecto ver cita de Flores Galindo en nota 9. 
cio privado, el cambio será negativo para la cultura indígena. Cuando estas manifestaciones se difunden en el espacio público, el cambio será positivo porque permitirá la revitalización de las expresiones culturales indígenas. En la revitalización de lo indígena será determinante el uso de los nuevos medios de comunicación, porque para Arguedas, las vías de transporte, la migración, la urbanización y los avances tecnológicos no eliminaban la cultura indígena (Rivera Andía, 2004: 284 y 85); desde su perspectiva, si bien transformaban lo indígena, esa transformación no suponía pérdida, era una suerte de transculturación. Kokotovic, que ha estudiado este tema con atención, señala que tanto mestizaje como transculturación son palabras sinónimas en los artículos arguedianos y "parecen significar cosas distintas en diferentes momentos". Algunas veces, estos términos pueden referirse a una influencia cultural mutua; pero, por lo general, estas categorías son utilizadas por Arguedas como "sinónimos de asimilación" (2006: 102). De esta suerte, la sinonimia trazada por Kokotovic se vuelve intercambiable y de golpe el mestizo arguediano oscila entre la transculturación y la asimilación. Los pasajes de los textos de Arguedas donde esta oscilación se manifiesta no hacen sino comprobar la observación de Kokotovic, pero el punto, me parece, puede plantearse de otra manera. Me refiero al hecho de que la asimilación cultural solo puede postularse de manera teórica como un horizonte de política cultural; en la práctica las relaciones entre las culturas siempre son dinámicas y los procesos son de ida y vuelta, aun cuando en algunos contextos son más de ida que de vuelta. Fernando Ortiz entendió muy bien la situación anterior al plantear el concepto de transculturación como un vocablo que comprendía mejor la complejidad del encuentro entre culturas, un proceso que suponía movimientos como la aculturación, entendida de manera radical como asimilación, pero fundamentalmente lo que él llamaba deculturación y neoculturación. La transculturación, dice, "no consiste solamente en adquirir una distinta cultura, que es lo que en rigor indica la voz angloamericana aculturation, sino que el proceso implica también necesariamente la pérdida o desarraigo de una cultura precedente, lo que pudiera decirse una parcial deculturación, y, además, significa la consiguiente creación de nuevos fenómenos culturales que pudieran denominarse de neoculturación" (1999: 83). Este tema es harto conocido, de modo que no voy a detenerme en ello. Lo importante para el caso de Arguedas es que en su trabajo de campo constata que la manera en que muchas formas del mundo andino han sobrevivido es bajo una dinámica que tenía la retórica de la asimilación, pero que en realidad era un proceso más bien transcultural. Fruto de ese proceso él identificará un actor: el mestizo, lo cual da un sentido histórico a sus investigaciones. En consecuencia, mestizaje y transculturación no comparten una relación de sinonimia para él, como ha indicado Kokotovic, sino de complementariedad. Mientras la lógica transcultu- 
rante define las relaciones entre la cultura europea y las indígenas; el mestizaje es un término con valor histórico que distingue una población caracterizada por un comportamiento cultural que negocia con el mundo europeo, en algunos casos bajo la forma de la asimilación, en otros, cuando las condiciones lo posibilitan como en el Valle del Mantaro, con una marcada prevalencia de las formas indígenas. Ambos casos, sin embargo, son los polos de un recorrido para nada excluyente, pero sí gradual, donde el mestizo es un personaje "que está desplegando una actividad poderosísima” (Arguedas, 1975: 2). De esta manera, como ha señalado Diana Taylor, Arguedas más que rescatar lo dominado, descentra lo dominante: "So rather than 'rescue' the dominated, Arguedas strives to decenter the dominant. Arguedas sabotages the hegemonic discourse by inserting the indigenous into the dominant culture" (1991: 96).

Una prueba actual de la vigencia del aporte de Arguedas en el campo de la etnología peruana es la reciente etnografía de Raúl R. Romero sobre el Valle del Mantaro. Al igual que Arguedas, y haciendo uso de sus fuentes, Romero profundiza la interpretación histórica que explica la excepcionalidad del área, la referencia a los Wankas, la posterior transformación del lugar en pueblo de indios y surgimiento como núcleo comercial que el área tuvo en el siglo XX y XXI (que es el punto de atención de su libro). Lo fundamental, sin embargo, para nuestro estudio, es la recuperación que hace del concepto de mestizo de Arguedas, mostrando su potencial operativo en el caso de su etnografía. Reconoce, por supuesto, que el concepto tiene connotaciones raciales y coloniales, a donde apuntaba la critica de Cornejo Polar, pero el le dará un sentido distinto: "El término mismo tiene fuertes connotaciones coloniales y raciales, pero las ciencias sociales han sostenido desde hace algún tiempo que el mestizaje en los andes sudamericanos no es un proceso racial, sino más bien cultural, el cual definimos aquí como la apropiación gradual de la modernidad por los campesinos indígenas andinos" (Romero, 2004: 45). La noción de campesinos indígenas andinos, para referirse a lo que Arguedas entendía como mestizo, expresa una mejor capacidad descriptiva, pero en lo fundamental sigue la propuesta de Arguedas:

En el contexto de una presencia violenta e impositiva del capitalismo moderno, vemos el mestizaje del Valle del Mantaro, siguiendo a Arguedas, como una iniciativa regional soberana, resultado de la clara determinación de los campesinos del valle de integrar sus economías domésticas en una creativa e imaginativa manera, para lo cual, como grupo, se apropian de las herramientas necesarias para negociar con el mercado en el mejor de los términos. De tal manera, el campesinado del valle acepta el bilingüismo, aprende español manteniendo el quechua, adopta los preceptos básicos del protocolo occidental auspiciado por el 
Estado e inscribe a sus niños en las escuelas públicas primarias. (Romero, 2004: 45)

La propuesta de Romero, que tiene un punto de partida no sólo histórico sino también teórico, en la obra de Arguedas, llegará a conclusiones de lo más interesantes. Por ejemplo, su propuesta de identidades múltiples (parecido al "Nosotros diverso" de Degregori), que es lo que está en el fondo de la idea de mestizaje de Arguedas, no un mestizaje armonizador, sino respetuoso de las tradiciones que reúne, y casi como una estrategia de existencia en el nivel personal, porque como nos recuerda Tarica, Arguedas enuncia desde el bilinguiismo. Este mestizaje que estuvo lleno de angustias para él, y que críticos literarios como Roberto Gonzalez Echevarría y Alberto Moreiras han leído, con ánimo apocalíptico y a la sombra de su suicidio, como una puesta en escena del fin del modelo de la antropología para la literatura, fue también la visión para Arguedas, en el caso del Valle del Mantaro, de una modernidad alternativa. La tan repetida frase del escritor al recibir el Premio Inca Garcilaso de la Vega: "Yo no soy un aculturado; yo soy un peruano que orgullosamente, como un demonio feliz, habla en cristiano y en indio, en español y en quechua" (1992: 257), que según la lectura de Flores Galindo, y también de Manrique, cuestionaría profundamente su propuesta de mestizaje (que ellos entienden como una utopía de la armonía en los términos de Riva Aguiero), no es más que la afirmación de que es posible una identidad múltiple -ni siquiera descentrada, fragmentada y migrante, como sugerirían los últimos trabajos de Cornejo Polar (1995: 11 y 12)- que el sujeto resuelve, diariamente, en su vivencia, tan dilthiana para Arguedas.

Quiero volver a la pregunta con que inicie estas páginas. ¿Cuál es la razón de que en su campo profesional Arguedas tenga un reconocimiento tardío y póstumo, y aún marginal en nuestros días? Un elemento de orden práctico, que se agrega a la discusión sobre el mestizaje que he presentado es la relación entre José Matos Mar y Arguedas. En el fondo, este parece un episodio de mezquindades personales, la típica historia de celos académicos: mientras Matos se hizo de un nombre dentro del naciente campo de la etnología, fue uno de los primeros estudiantes de Valcárcel en graduarse; Arguedas llega al campo con un capital simbólico forjado por sus novelas y su labor como periodista, folklorista y animador cultural de la peña Pancho Fierro. Un testigo de la época, Alejandro Ortiz Rescaniere, quien fuera estudiante de ambos, nos informa:

[...] notaba que Matos tomaba cierta distancia con los alumnos que estaban vinculados a Arguedas. Matos y Arguedas eran dos personalidades, de estilos y de formas diferentes. Matos, más científico y frio. Arguedas, más emotivo, afectivo. Sí, notaba una cierta rivalidad. Me 
acuerdo bien, cuando [Arguedas] fue a visitarme a Francia, me dijo que Matos era un posero, que vendía su imagen de indio. Creo que detrás de todo ello se escondía un cierto celo profesional de parte de Arguedas. Por el lado de Matos habría, quien sabe, algo de celos por el éxito literario de Arguedas; porque no podía sentir rivalidad alguna en el aspecto antropológico, pues Matos se sentía, al respecto, superior a Arguedas [...] Matos no necesitaba afirmarse en nada. Era un hombre seguro de sí mismo. Era un profesional de acción, hábil en la tarea de conseguir fondos, proyectos, becas en el extranjero. Era fácil para los estudiantes identificarse con él. (1996: 188 y 89).

No es difícil imaginar, como sugiere el testimonio de Ortiz Rescaniere, que en un ambiente dominado por la teoría del desarrollo y la antropología aplicada, del cual Matos Mar fue el hombre de avanzada, las posiciones heterodoxas de Arguedas fueran marginalizadas. El fracaso de Arguedas en establecer un diálogo con sus colegas, será aún más elocuente en la célebre mesa redonda sobre Todas las sangres, donde los científicos sociales de entonces, entre ellos Matos Mar, fueron despiadados con el escritor (y supongo que con nosotros, su publico actual) al exhibir sus incapacidades para leer literatura. ${ }^{13}$

A la luz de este último encuentro con sus colegas de ciencias sociales, la marginalización del trabajo etnográfico de Arguedas tiene más sentido. Y mientras que su literatura cobraba una significación trascendente para el Perú y los estudios latinomericanos, gracias a la lectura atenta de críticos extranjeros como Rama, Martín Lienhard, Rowe o peruanos como Cornejo Polar y Alberto Escobar; su muerte lo terminó de alejar de la lista de aportantes a la etnografía peruana de las décadas de 1970 y 1980 . Pero es gracias a su literatura, y las lecturas que desde la crítica literaria se ha hecho de la obra de Arguedas, que este autor retorna, como todo lo que está mal enterrado, y revela una

13 Entre los científicos sociales estuvieron, además de Matos Mar, Jorge Bravo Bresani, Henry Favre y Aníbal Quijano. Por la literatura solo Alberto Escobar destacó positivamente el trabajo con la palabra de Arguedas. Sebastián Salazar Bondy y José Miguel Oviedo tuvieron posturas parecidas a las del primer grupo. Para Melisa Moore, lo que se disputaba en la discusión era la capacidad de la novela para representar la realidad peruana (2005, p. 269). Arguedas se defendió diciendo que no pretendía escribir un tratado sociológico, "dando a entender que su obra podía ser vista más bien como un texto etnográfico [...] A la luz de las recientes teorías elaboradas por James Clifford [...] afirmaciones semejantes no serían motivo de sorpresa o reacción, pero hace casi cuarenta años ello dio lugar a que se condenara a Arguedas en forma vociferante" (Moore 2005: 269). La mejor respuesta de Arguedas, sin embargo, ha sido ese poema bellísimo, escrito en quechua y español, titulado "Llamado a algunos doctores", dedicado por cierto a otros doctores, John Murra y Carlos Cueto Fernandini. El poema apareció en el suplemento El Dominical del diario El Comercio el 3 de julio de 1966. Sobre el aporte y la influencia de Arguedas como poeta en la poesía peruana contemporánea resulta imprescindible el primer capítulo de Poéticas del flujo de José Antonio Mazzotti (2002). 
verdad al campo de los estudios etnológicos. ${ }^{14} \mathrm{~A}$ juzgar por el libro de Romero, tal retorno es potente. A la luz de lo expuesto, me gustaría insistir, a modo de conclusión, en que si bien la intervención de Arguedas en el campo del pensamiento sobre la cultura peruana ha sido accidentada, no por ello ha carecido de razón. Su idea de mestizaje, que imaginaba una modernidad alternativa, es realidad cotidiana en el Perú actual. ${ }^{15}$

\section{Referencias bibliográficas}

Archibald, P. (1998) "Andean anthropology in the era of development theory: The work of José María Arguedas". En José María Arguedas. Reconsiderations for Latin American Cultural Studies, eds. C.A. Sandoval \& S. M. Brocheto, Ohio University, Athens, pp.3-34.

Arguedas, J. M. (1992) El zorro de arriba y el zorro de abajo. México: Archivos.

Arguedas, J. M. (1989) Indios, mestizos y señores. Horizonte: Lima.

Arguedas J. M. (1987) Formación de una cultura nacional indoamericana. México: Siglo XXI. Arguedas, J. M. (1968) Las comunidades de España y el Perú.Lima: UNMSM.

Arguedas, J. M. (1964) "Prólogo". En Estudios sobre la cultura actual del Perú. Lima: UNMSM; pp. 5-7.

Ávila Molero, J. (2000) "Los dilemas del desarrollo: antropología y promoción en el Perú”, En_No hay país más diverso. Compendio de antropología peruana, Ed C. I. Degregori. Lima: Red; pp. 413-442.

Bernabé, M. (2005) “José María Arguedas traductor”. En José María Arguedas: Hacia una poética migrante, Ed Sergio Franco, Pittsburgh IILI; pp. 371-88.

Burga, M. (2001) "Historia y antropología en la historiografía peruana (1987-1998)". En Muchas hispanoaméricas. Antropología, historia y enfoques culturales en los estudios latinoamericanos, eds T. Krüggeler \& U. Mücke, Madrid: Iberoamericana \& Vervuert; pp. 25-47.

Cornejo Polar, A (1995) "Condición migrante y representatividad social: El caso de Arguedas”. En Amor y fuego. José María Arguedas, 25 años después, Eds Maruja. Martínez \& Nelson. Manrique. Lima: Desco; pp. 3-14.

Cornejo Polar, A. (1994) Escribir en el aire. Ensayo sobre la heterogeneidad socio-cultural en las literaturas andinas. Lima: Horizonte.

Cornejo Polar, A. (1993) "El discurso de la armonía imposible (El Inca Garcilaso de la Vega: discurso y recepción social)" en Revista de Crítica Literaria Latinoamericana, 38; pp. 73-80.

14 No podemos dejar de mencionar aquí el trabajo tenaz, y ahora exitoso, por difundir su obra que tanto su discípulo Ortiz Rescaniere, como María del Carmen Pinilla han hecho desde el campo de la antropología.

15 Una versión de este texto se publicó en inglés en Latin American \& Caribbean Ethnic Studies 4.2. 
Degregori, C. I. (2000) "Panorama de la antropología en el Perú: del estudio del Otro a la construcción de un Nosotros diverso". En No hay país más diverso. Compendio de antropología peruana. Lima: Red; pp.20-73.

Del Pino, F. (1995) “Arguedas en España o la condición mestiza de la antropología”. En Amor y fuego. José María Arguedas, 25 años después, Eds. Maruja. Martínez \& Nelson Manrique. Lima: Desco; pp. 23-55.

De la Cadena, M. (2000) Indigenous Mestizos. The Politics of Race and Culture in Cusco, Peru, 1919-1991. Duke UP, Durham \& London.

Escajadillo, T. (1994), La narrativa indigenista peruana. Lima: Amaru.

Flores Galindo, A. (1992) Dos ensayos sobre José María Arguedas. Lima: SUR.

Flores Galindo, A. (1988) Buscando un Inca. Lima: Horizonte.

González Echevarria, R. (1998) Myth and Archive. A Theory of Latin American Narrative, Duke UP, Durhan \& London.

Kokotovic, M. (2006) La modernidad andina en la narrativa peruana: conflicto social y transculturación. Lima: Latinoamericana editores.

Krüggeler, T. \& Müicke, U. (2001) “Introducción: historia y antropología en los estudios latinoamericanos”. En Muchas hispanoaméricas. Antropología, historia y enfoques culturales en los estudios latinoamericanos, eds T. Krüggeler \& U. Mücke, Madrid: Iberoamericana \& Vervuert; pp.9-24.

Lauer, M. (1996) Andes imaginarios. Discursos del indigenismo 2, Lima: CBC \& SUR.

Manrique, N. (1999) La piel y la pluma. Escritos sobre literatura, etnicidad y racismo.Lima: SUR.

Manrique, N. “José María Arguedas, la cuestión del mestizaje”. En Amor y fuego. José María Arguedas, 25 años después, Eds Maruja Martínez \& Nelson Manrique.Lima: Desco; pp. 77-89.

Mariategui, J. C. (1979), Siete ensayos de interpretación de la realidad peruana. Caracas: Biblioteca Ayacucho.

Mazzotti, J. A. (2002) Poéticas del flujo. Migración y violencia verbales en el Perú de los 80, Lima: Fondo Editorial del Congreso del Perú.

Montoya, R. (1991) “Antropología y política”. En José María Arguedas, veinte años después: huellas y horizontes. 1969-1989. Lima: Escuela de Antropología UNMSM.

Moore, M. (2005) "Encuentros y desencuentros de la novela y las ciencias sociales en el Perú: repensando Todas las sangres de José María Arguedas”. En José María Arguedas: Hacia una poética migrante, Ed Sergio Franco. Pittsburgh IILI; pp. 267-284.

Moreiras, A. (1997) “José María Arguedas y el fin de la transculturación”. En Ángel Rama y los estudios latinoamericanos, ed M. Moraña. Pittsburgh: IILI; pp. 213-231.

Osterling, J. \& Martínez, H. (1985) "Apuntes para una historia de la antropología social peruana: décadas de 1940-1980”. En La antropología en el Perú, ed H. Rodríguez Pastor, Lima: Concytec; pp. 35-68. 
Ortiz, F. (1999) Contrapunteo cubano del azúcar y el tabaco (Advertencia de sus contrastes agrarios, económicos, históricos y sociales, su etnografía y su transculturación). Madrid: EditoCubaEspana.

Ortiz Rescaniere, A. (editor) (1996) José María Arguedas. Recuerdos de una amistad. Lima: Fondo Editorial PUCP.

Portocarrero, G. (1993) Racismo y mestizaje. Lima: SUR.

Rama, A. (1987) “Introducción.”. En J. M. Arguedas, Formación de una cultura nacional indoamericana. México:Siglo XXI; pp. X-XXVII.

Rivera Andía, J. J. (2004) "La pasión y los medios. Aproximaciones a la obra etnológica de Arguedas y al concepto de 'cambio cultural' en la antropología peruana”. En Arguedas en el Valle del Mantaro, ed C.M. Pinilla. Lima: PUPCP; pp. 195-301.

Romero, R. (2004) Identidades múltiples. Memoria, modernidad y cultura popular en el valle del Mantaro. Lima: Fondo Editorial del Congreso del Perú.

Rowe, William, (1979) Mito e ideología en la obra de José María Arguedas. Lima: INC.

Salomon, F. (1982), "Andean Ethnology in the 1970s: A Retrospective" en Latin American Research Review vol. 17, 2; pp. 75-128.

Tarica, Estelle (2005) “El ‘decir limpio’ de Arguedas: la voz bilingüe, 1940-1958”. En José María Arguedas: Hacia una poética migrante, ed S. Franco. Pittsburgh: IILI; pp. 15-30.

Taylor, D. (1992) “Transculturating Transculturation” en Performing Arts Journal, Vol. 13, 2; pp. 90-104.

Tomoeda, H. (2006) "Inkarrí en La Habana: discurso indigenista en torno a un mito indígena”. En Desde el exterior. El Perú y sus estudiosos. Tercer Congreso Internacional de Peruanistas. Nagoya, 2005, eds L. Millones \& T. Kato, Lima:Fondo Universidad San Marcos; pp. 167-90.

Valcárcel, L. E. (1985) “Inicios de la antropología en el Perú”. En La antropología en el Perú, ed H. Rodríguez Pastor, Lima: Concytec; pp.15-28.

Valcárcel, L. E. (1972) Tempestad en los andes. Lima: Universo. 
Enrique E. Cortez 